\title{
PLASMA EXPANSION IN THE GEOMETRY OF A COLLAPSING STAR
}

\author{
R. RUFFINI, L. VITAGLIANO AND S.-S. XUE
}

\begin{abstract}
We describe the evolution of an electron-positron-photon plasma created by Sauter-Heisenberg-Euler-Schwinger mechanism around a collapsing charged star core in the Reissner-Nordström geometry external to the core, in view of the application in the framework of the EMBH theory for gamma ray bursts.
\end{abstract}

\section{Introduction}

In 1975 , following the work on the energetics of black holes, ${ }^{1}$ Damour and Ruffini $^{2}$ pointed out the existence of the vacuum polarization process $\grave{a}$ là Sauter-Heisenberg-Euler-Schwinger ${ }^{3,4}$ around black holes endowed with electromagnetic structure (EMBHs), whose electric field strength exceeds the Schwinger critical value $\mathcal{E}_{\mathrm{c}}=\frac{m_{e}^{2} c^{3}}{e \hbar}$, where $c$ is the speed of light, $e$ and $m_{e}$ are electron charge and mass respectively. Damour and Ruffini gave reasons to believe that this process is almost reversible in the sense introduced by Christodoulou and Ruffini ${ }^{1}$ and that it extracts the mass energy of an EMBH very efficiently: this have been proved in Ref. 5. The vacuum polarization process around an EMBH offered a natural mechanism for explaining the Gamma Ray Bursts (GRBs), just discovered at the time. Moreover the mechanism had a most peculiar prediction: the characteristic energetics of the burst should be of the order of $10^{54} \mathrm{ergs}$; while nothing at the time was known about either the distances or the energetics of GRBs.

More recently, after the discovery of the afterglow of GRBs and their cosmological distance, the idea by Damour and Ruffini has been reconsidered in Refs. 6-9 where the EMBH model for GRBs is developed. The evidence is now that through the observations of GRBs we are witnessing the formation of an EMBH and therefore are following the process of gravitational collapse in real time. Even more importantly, the tremendous energies involved in the energetics of these sources have their origin in the extractable energy of black holes.

Various models have been proposed in order to extract the rotational energy of black holes by processes of relativistic magnetohydrodynamics 
(see, e.g., Ref. 10). It should be expected, however, that these processes are relevant over the long time scales characteristic of accretion processes. In the present case of GRBs a sudden mechanism appears to be at work on time scales of the order of few seconds or shorter and they are naturally explained by the vacuum polarization process introduced in Ref. 2 .

All considerations on the electric charge of stars have been traditionally directed towards the presence of a net charge on the star surface in a steady state condition, from the classic work by Shvartsman ${ }^{11}$ all the way to the fundamental book by Punsly. ${ }^{12}$ The charge separation can occur in stars endowed with rotation and magnetic field and surrounded by plasma, as in the case of Goldreich and Julian, ${ }^{13}$ or in the case of absence of both magnetic field and rotation, the electrostatic processes can be related to the depth of the gravitational well, as in the treatment of Shvartsman. ${ }^{11}$ However, in neither case is it possible to reach the condition of the overcritical field needed for pair creation.

The basic new conceptual point is that GRBs are the most violent transient phenomenon in the universe and therefore in order to realize the condition for their occurrence, one must look at a transient phenomenon. We propose as a candidate the most transient phenomenon possibly occurring in the life of a star: the gravitational collapse. The condition for the creation of the supercritical electromagnetic field required in the Damour and Ruffini work has to be achieved during the process of gravitational collapse which lasts less than $\sim 30$ seconds for a mass of $10 M_{\odot}$ and the relevant part of the process may be as short as $10^{-2}$ or even $10^{-3}$ seconds. It is appropriate to consider a numerical example here ${ }^{14}$ (see Fig. 1). We compare and contrast the gravitational collapse of a star in the two limiting cases in which its core of $M=3 M_{\odot}$ and radius $R=R_{\odot}$ is either endowed with rotation or with electromagnetic structure. The two possible outcomes of the process of gravitational collapse are considered: either a neutron star of radius of $10 \mathrm{~km}$ or a black hole.

In the case of rotation the core has been assumed to have a rotational period of $\sim 15$ days. For such an initial configuration we have:

$$
E_{\text {rot }} \simeq 7 \times 10^{-12} E_{\text {tot }} \ll\left|E_{\text {grav }}\right| \simeq 6 \times 10^{-6} E_{\text {tot }} \ll E_{\text {bar }} \simeq 4.4 \times 10^{5} \mathrm{~cm} .
$$

In the collapse to a neutron star we have:

$$
E_{\text {rot }} \simeq 0.01 E_{\text {tot }} \ll\left|E_{\text {grav }}\right| \simeq 0.1 E_{\text {tot }} \ll E_{\text {bar }} \simeq 4.4 \times 10^{5} \mathrm{~cm} .
$$

The very large increase in the rotational energy is clearly due to the process of gravitational collapse: such a storage of rotational energy is the well 


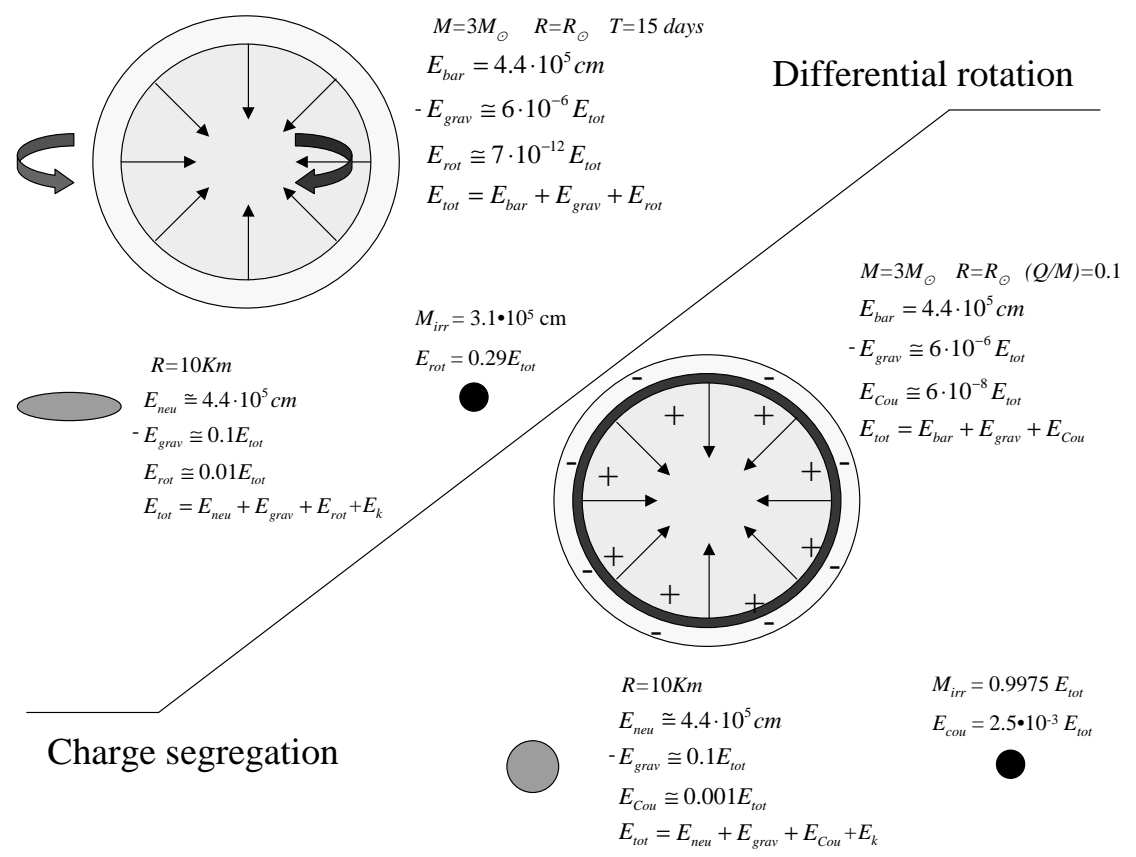

Figure 1. Comparing and contrasting gravitational collapse to a neutron star and to a black hole for a star core endowed with rotation or electromagnetic structure. Reproduced from Ref. [14] with the kind permission of the author.

known process explaining the pulsar phenomena. The collapse to a black hole has been estimated assuming the mass-energy formula. ${ }^{1}$ The overall energetics, for the chosen set of parameters, leads to a solution corresponding to an extreme black hole, for which in principle $29 \%$ of the energy is extractable.

The similar process in the electromagnetic case starts from an initial neutral star with a magnetosphere oppositely charged from a core with

$$
\frac{Q}{M \sqrt{G}}=0.1
$$

Let us first evaluate the amount of polarization needed in order to reach the above relativistic condition. Recalling that the charge to mass ratio of a proton is $q_{p} /\left(m_{p} \sqrt{G}\right)=1.1 \times 10^{18}$, it is enough to have an excess of one quantum of charge every $10^{19}$ nucleons in the core of the collapsing star to obtain such an EMBH after the occurrence of the gravitational collapse. Physically this means that we are dealing with a process of charge segregation between the core and the outer part of the star which has the opposite sign of net charge in order to enforce the overall charge neutrality 
condition.

We then have:

$$
E_{\text {cou }} \simeq 6 \times 10^{-8} E_{\text {tot }} \ll\left|E_{\text {grav }}\right| \simeq 6 \times 10^{-6} E_{\text {tot }} \ll E_{\text {bar }} \simeq 4.4 \times 10^{5} \mathrm{~cm} .
$$

In the collapse to the neutron star configuration we have:

$$
E_{\text {Cou }} \simeq 0.001 E_{\text {tot }} \ll\left|E_{\text {grav }}\right| \simeq 0.1 E_{\text {tot }} \ll E_{\text {bar }} \simeq 4.4 \times 10^{5} \mathrm{~cm} .
$$

Once again, the amplification of the electromagnetic energy is due to the process of gravitational collapse. Again, assuming the mass-energy formula, the collapse to a black hole for the chosen set of parameters leads to:

$$
M_{\mathrm{ir}}=0.9975 E_{\text {tot }}, \quad E_{\text {Cou }}=2.5 \times 10^{-3} E_{\text {tot }} .
$$

It is during such a process of gravitational collapse to an EMBH that the overcritical field is reached.

The process of charge segregation between the inner core and the oppositely charged outer shell is likely due to the combined effects of rotation and magnetic fields in the earliest phases of the gravitational collapse of the progenitor star or to a process of ionization. In the following we will forget about the outer shell and will treat the inner core as an electrically charged collapsing star.

\section{Energy extraction from a supercritical EMBH}

We know from the Christodoulou-Ruffini mass formula ${ }^{1}$ that the mass energy of an EMBH is the sum of the irreducible mass and the electromagnetic energy:

$$
M=M_{\mathrm{ir}}+\frac{Q^{2}}{2 r_{+}},
$$

where $Q$ is the charge and $r_{+}$is the radius of the horizon. Moreover in Ref. 5 it is shown that the electromagnetic energy $\frac{Q^{2}}{2 r_{+}}$is stored throughout the region external to the EMBH and can be extracted. If the condition

$$
\frac{Q}{r_{+}^{2}} \geq \mathcal{E}_{\mathrm{c}}
$$

is fulfilled the leading extraction process is a collective process based on the electron-positron plasma generated by Schwinger mechanism in the supercritical electric field of the EMBH. ${ }^{5}$ The condition (8) implies

$$
\frac{G M / c^{2}}{\lambda_{\mathrm{C}}}\left(\frac{e}{\sqrt{G} m_{e}}\right)^{-1} \simeq 2 \cdot 10^{-6} \frac{M}{M_{\odot}} \leq \xi \leq 1
$$


and therefore this vacuum polarization process can occur only for an EMBH with mass smaller than $2 \cdot 10^{6} M_{\odot}$. The electron-positron pairs are produced in the dyadosphere of the EMBH, ${ }^{6}$ the spherical region whose radius $r_{\mathrm{ds}}$ satisfies $\mathcal{E}_{\mathrm{c}} \equiv \frac{Q}{r_{\mathrm{ds}}^{2}}$. We have

$$
r_{\mathrm{ds}}=\sqrt{\frac{e Q \hbar}{m_{e}^{2} c^{3}}} .
$$

The number of particles created is ${ }^{6}$

$$
N_{\mathrm{ds}}=\frac{1}{3}\left(\frac{r_{\mathrm{ds}}}{\lambda_{\mathrm{C}}}\right)\left(1-\frac{r_{+}}{r_{\mathrm{ds}}}\right)\left[4+\frac{r_{+}}{r_{\mathrm{ds}}}+\left(\frac{r_{+}}{r_{\mathrm{ds}}}\right)^{2}\right] \frac{Q}{e} \simeq \frac{4}{3}\left(\frac{r_{\mathrm{ds}}}{\lambda_{\mathrm{C}}}\right) \frac{Q}{e} .
$$

The total energy stored in the dyadosphere is ${ }^{6}$

$$
E_{\mathrm{ds}}^{\mathrm{tot}}=\left(1-\frac{r_{+}}{r_{\mathrm{ds}}}\right)\left[1-\left(\frac{r_{+}}{r_{\mathrm{ds}}}\right)^{4}\right] \frac{Q^{2}}{2 r_{+}} \simeq \frac{Q^{2}}{2 r_{+}} .
$$

The mean energy per particle produced in the dyadosphere $\langle E\rangle_{\mathrm{ds}}=\frac{E_{\mathrm{ds}}^{\mathrm{tot}}}{N_{\mathrm{ds}}}$ is then

$$
\langle E\rangle_{\mathrm{ds}}=\frac{3}{2} \frac{1-\left(\frac{r_{+}}{r_{\mathrm{ds}}}\right)^{4}}{4+\frac{r_{+}}{r_{\mathrm{ds}}}+\left(\frac{r_{+}}{r_{\mathrm{ds}}}\right)^{2}}\left(\frac{\lambda_{\mathrm{C}}}{r_{\mathrm{ds}}}\right) \frac{Q e}{r_{+}} \simeq \frac{3}{8}\left(\frac{\lambda_{\mathrm{C}}}{r_{\mathrm{ds}}}\right) \frac{Q e}{r_{+}},
$$

which can be rewritten as

$$
\langle E\rangle_{\mathrm{ds}} \simeq \frac{3}{8}\left(\frac{r_{\mathrm{ds}}}{r_{+}}\right) m_{e} c^{2} \sim \sqrt{\frac{\xi}{M / M_{\odot}}} 10^{5} \mathrm{keV} .
$$

Such a process of vacuum polarization, occurring not at the horizon but in the extended dyadosphere region $\left(r_{+} \leq r \leq r_{\mathrm{ds}}\right)$ around an EMBH, has been observed to reach the maximum efficiency limit of $50 \%$ of the total mass-energy for an extreme EMBH (see e.g. Ref. 6). As discussed in Ref. 5 the $e^{+} e^{-}$creation process occurs at the expense of the Coulomb energy and does not affect the irreducible mass, which does not depend of the electromagnetic energy. In this sense, $\delta M_{\mathrm{ir}}=0$ and the transformation is fully reversible.

\section{The EMBH Theory}

In a series of papers, ${ }^{6-9}$ Ruffini and collaborators have developed the EMBH theory for GRBs, which has the advantage, despite its simplicity, that all eras following the process of gravitational collapse to the EMBH are described by precise field equations which can then be numerically integrated. 
Starting from the vacuum polarization process à là Sauter-HeisenbergEuler-Schwinger in the overcritical field of an EMBH first computed in Ref. 2, Ruffini et al. developed the dyadosphere concept. ${ }^{6}$

The dynamics of the $e^{+} e^{-}$-pairs and electromagnetic radiation of the plasma generated in the dyadosphere propagating away from the EMBH in a sharp pulse (PEM pulse) has been studied by the Rome group and validated by the numerical codes developed at Livermore Lab. ${ }^{15,16}$

The collision of the still optically thick $e^{+} e^{-}$-pairs and electromagnetic radiation plasma with the baryonic matter of the remnant of the progenitor star has been again studied by the Rome group and validated by the Livermore Lab codes. ${ }^{15,16}$ The further evolution of the sharp pulse of pairs, electromagnetic radiation and baryons (PEMB pulse) has been followed for increasing values of the gamma factor until the condition of transparency is reached. ${ }^{17}$

As this PEMB pulse reaches transparency the proper GRB (P-GRB) is emitted $^{8}$ and a pulse of accelerated baryonic matter (the ABM pulse) is injected into the interstellar medium (ISM) giving rise to an afterglow. Thus in GRBs we can distinguish an injector phase and a beam-target phase. The injector phase includes the process of gravitational collapse, the formation of the dyadosphere, as well as the PEM pulse, the engulfment of the baryonic matter of the remnant and the PEMB pulse. The injector phase terminates with the P-GRB emission. The beam-target phase addresses the interaction of the ABM pulse, namely the beam generated during the injection phase, with the ISM as the target. It gives rise to the E-APE (Extended Afterglow Peak Emission) and the decaying part of the afterglow. The existence of both the P-GRB and the E-Ape is shown in Fig. 2, where the fit of observational data relative to GRB 991216 within the EMBH theory is reported.

\section{Gravitational Collapse of an Electrically Charged Core: Formation of Dyadosphere}

We now turn to the details of the formation of dyadosphere. If the electric field of a charged star core is stable against vacuum polarization during the gravitational collapse, ${ }^{18}$ then an enormous amount of pairs can be created by Schwinger mechanism. Moreover the pairs thermalize to a positronselectrons-photons plasma configuration (see Refs. 6, 19, 20). Such a plasma undergoes a relativistic expansion. The evolution of the system and the details of GRB emission, along the lines summarized in the previous section, 


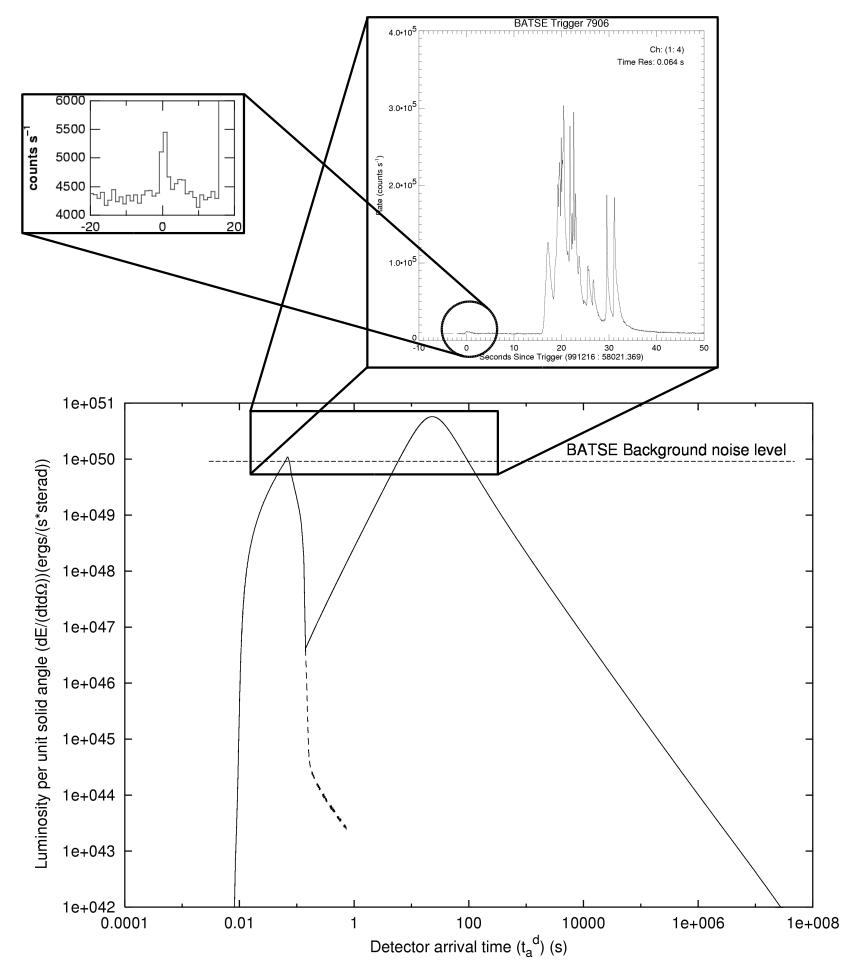

Figure 2. The overall description of the EMBH theory applied to GRB 991216. The BATSE noise threshold is represented and the observations both of the P-GRB and of the E-APE are clearly shown in the subpanels. The continuos line in the picture represents the theoretical prediction of the EMBH model.

were described in Refs. $6-8,15-17$. In the latter papers the time scale of the gravitational collapse is neglected with respect to the hydrodynamic time scale. In this paper we relax this approximation: our main aim is to describe how the plasma expansion occurs during the gravitational collapse. In a forthcoming paper ${ }^{18}$ we will discuss how the expansion is affected by the strong gravitational field near the horizon of the forming EMBH.

In Refs. 20 and 21 it was suggested that the exact solution of EinsteinMaxwell equations describing the gravitational collapse of a thin charged shell can be used as an analytical model for the gravitational collapse of a charged core. First we briefly review some of the results of Ref. 21. The region of space-time external to the collasping core is Reissner-Nordström 
with line element, in Schwarzschild like coordinates $(t, r, \theta, \phi)$,

$$
d s^{2}=-\alpha^{2} d t^{2}+\alpha^{-2} d r^{2}+r^{2} d \Omega^{2},
$$

where $d \Omega^{2}=d \theta^{2}+\sin ^{2} \theta d \phi^{2}, \alpha^{2}=\alpha^{2}(r)=1-\frac{2 M}{r}+\frac{Q^{2}}{r^{2}}, M$ is the total energy of the core as measured at infinity and $Q$ is its total charge. Let us label with $r_{0}$ and $t_{0}$ the radial and time-like coordinates of the shell, then the electromagnetic field strength on the surface of the core is $\mathcal{E}=\mathcal{E}\left(r_{0}\right)=\frac{Q}{r_{0}^{2}}$ and the equation of core's collapse is ${ }^{21}$

$$
\frac{d r_{0}}{d t_{0}}=-\frac{\alpha^{2}\left(r_{0}\right)}{H\left(r_{0}\right)} \sqrt{H^{2}\left(r_{0}\right)-\alpha^{2}\left(r_{0}\right)}
$$

where $H\left(r_{0}\right)=\frac{M}{M_{0}}-\frac{M_{0}^{2}+Q^{2}}{2 M_{0} r_{0}}$ and $M_{0}$ is the rest mass of the shell. The analytical solution of Eq. (16) was found in Ref. 21 in the form

$$
t_{0}=t_{0}\left(r_{0}\right) \text {. }
$$

Dyadosphere is formed since the instant $t_{\mathrm{ds}}=t_{0}\left(r_{\mathrm{ds}}\right)$ when $\mathcal{E}=\mathcal{E}_{\mathrm{c}}$. In the following we put $t_{\mathrm{ds}}=0$.

\section{Formation of $e^{+} e^{-}$Pairs around a Collapsing Charged Core}

For $t<t_{\mathrm{ds}}, \mathcal{E}<\mathcal{E}_{\mathrm{c}}$ and the Schwinger process of $e^{+} e^{-}$pairs creation is exponentially suppressed. For $t>t_{\mathrm{ds}}$ the Schwinger process becomes relevant and $e^{+} e^{-}$pairs are created. As shown in Refs. 19,20 the pairs created at radius $r_{0}<r_{\text {ds }}$ oscillate with ultrarelativistic velocity and partially annihilate into photons. At the same time the electric field oscillates around zero and the amplitude of such oscillations decreases in time: in a time of the order of $10^{2}-10^{4} \hbar / m_{e} c^{2}$ the electric field is effectively screened to about the critical value; more precisely, the average of the electric field $\mathcal{E}$ over one period of oscillation is 0 , but the average of $\mathcal{E}^{2}$ is of the order of $\mathcal{E}_{c}^{2}$. As a result an energy density has been deposited ${ }^{5}$ on the pairs and the photons given by

$$
\epsilon_{0}\left(r_{0}\right)=\frac{1}{8 \pi}\left[\mathcal{E}^{2}\left(r_{0}\right)-\mathcal{E}_{c}^{2}\right]=\frac{\mathcal{E}_{c}^{2}}{8 \pi}\left[\left(\frac{r_{\mathrm{ds}}}{r_{0}}\right)^{4}-1\right] .
$$

The pairs and the photons are expected to thermalize ${ }^{6,19,20}$, to an $e^{+} e^{-} \gamma$ plasma equilibrium configuration:

$$
n_{e^{+}}=n_{e^{-}} \simeq n_{\gamma}=n_{0},
$$


(where $n_{\bullet}$ is the proper number density of particles of type $\bullet$ ), and reach an average temperature $T_{0}$ such that

$$
\epsilon\left(T_{0}\right) \equiv \epsilon_{\gamma}\left(T_{0}\right)+\epsilon_{e^{+}}\left(T_{0}\right)+\epsilon_{e^{-}}\left(T_{0}\right)=\epsilon_{0}
$$

here $\epsilon_{\bullet}(T)$ is the equilibrium proper energy density at temperature $T$ for the species $\bullet$. Then $n_{e^{ \pm}}\left(n_{\gamma}\right)$ are given by Fermi (Bose) integrals once the temperature $T_{0}$ is known.

\section{Plasma's Expansion}

The highly energetic plasma so formed undergoes a relativistic expansion. As will be shown, the expansion (hydrodynamic) time-scale is much bigger than both the pair creation and the thermalization time-scales, then the process can be described as follows: at any time $t_{0}$ it begins to expand a slab of plasma of thickness $\Delta l=\alpha^{-1} \Delta r$ (as measured by static observers) produced at radius $r_{0}=r_{0}\left(t_{0}\right) . \Delta l$ can be chosen very small in comparison with $r_{\mathrm{ds}}$ so that, in particular, the temperature $T$ is approximately constant in the slab. Moreover $\Delta l$ has to be much bigger than the quantum length scale $\left(\sim \hbar / m_{e} c\right)$.

We can follow the expansion of each slab of plasma by using conservation of energy and number of particles. Note that Eqs. (20) and (19) provide initial data for the problem of the expansion. We describe the expansion of a single slab using the following approximations:

(1) the geometry in which the expansion occurs is Reissner-Nordström with line-element given by (15). In particular we will denote by $\xi^{a}$ the static vector field normalized at unity at spatial infinity, and by $\left\{\Sigma_{t}\right\}_{t}$ the family of space-like hypersurfaces orthogonal to $\xi^{a}(t$ being the Killing time);

(2) the plasma is assumed to be a neutral perfect fluid characterized by proper energy density $\epsilon$, proper pressure $p$, proper particle (electrons, positrons and photons) number density $n$ and 4-velocity $u^{a}$;

(3) at any instant, electrons, positrons and photons in a single slab are assumed to be at thermal equilibrium with temperature $T$, possibly different from slab to slab. The slabs are uncorrelated in the sense that they do not share energy nor particles. In other words the expansion of each slab is adiabatic; this will be checked a posteriori (see also Ref. 16);

(4) the thickness $\Delta l=\alpha^{-1} \Delta r$ of a slab as measured by static observers is constant. 


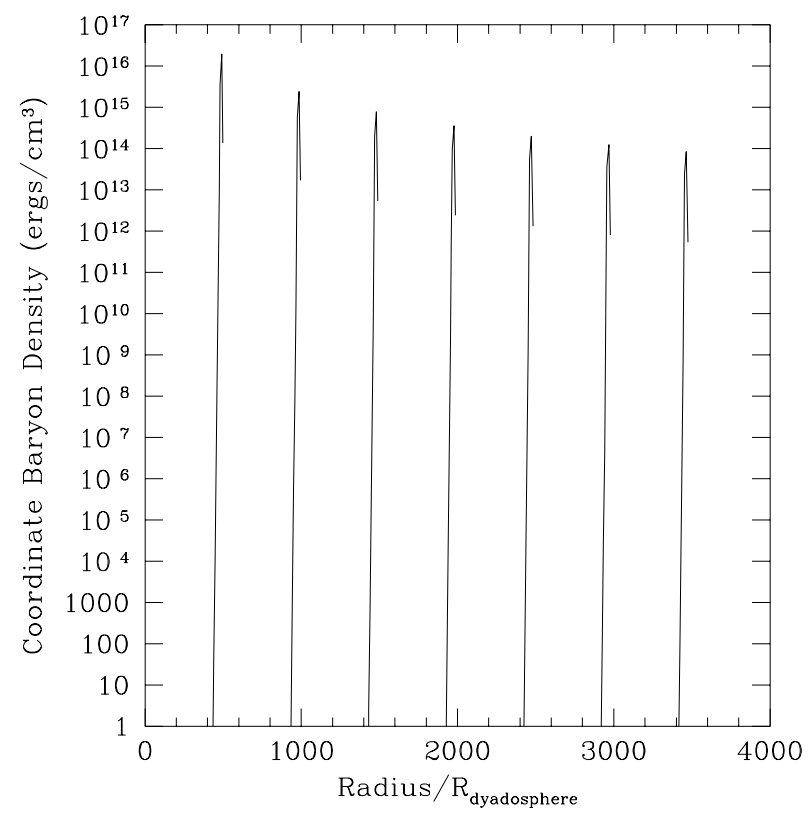

Figure 3. A sequence of snapshots of coordinate baryon energy density is shown from the numerical solution of partial differential continuity equations. This run correspond to an EMBH of mass $M=10^{3} M_{\odot}$ and charge to mass ratio $\xi \equiv Q / M=0.1$.

The last approximation is justified by the result in Ref. 16, where it is shown, by numerical integration of the partial differential continuity equations, that the baryon energy density of an expanding slab of plasma enriched with nucleons from the remnant of the progenitor star is localized in a region of constant thickness (see Fig. 3).

Given the above assumptions, both the energy momentum-tensor $T^{a b}=$ $(\epsilon+p) u^{a} u^{b}+p g^{a b}$ and the electron (positron) -number current $n_{e}^{a}=n_{e} u^{a}$ are conserved:

$$
\begin{aligned}
\nabla_{b} T^{a b} & =0, \\
\nabla_{b} n_{e}^{b} & =0 .
\end{aligned}
$$

In particular, using assumption (4) one can reduce the partial differential continuity equations (21) and (22) to ordinary differential equations for the radial coordinate $r$ and the temperature $T$ of the single slab as functions of time (see Ref. 16). The equation of motion of a single slab can be 
numerically integrated with initial conditions

$$
\begin{aligned}
r\left(t_{0}\right) & =r_{0}, \\
\left.\frac{d r}{d t}\right|_{t=t_{0}} & =0, \\
T\left(t_{0}\right) & =T_{0} .
\end{aligned}
$$

The overall motion of the plasma is the superposition of motions of single shells. The typical plasma expansion curves are shown in Fig. 4 from the numerical integration of the equations of motion.

The curvature of space-time strongly affects the motion of plasma in the vicinity of the EMBH horizon and in turn the phenomenology of the GRB. We discuss these issues in a forthcoming paper. ${ }^{18}$

\section{References}

1. D. Christodoulou and R. Ruffini, Phys. Rev. D4, 3552 (1971).

2. T. Damour, R. Ruffini, Phys. Rev. Lett. 35, 463 (1975).

3. W. Heisenberg and H. Euler, Zeits. Phys. 98, 714 (1935).

4. J. Schwinger, Phys. Rev. 98, 714 (1951).

5. R. Ruffini, L. Vitagliano, Phys. Lett. B 545 (2002) 233.

6. G. Preparata, R. Ruffini and S.-S. Xue, A\&A 338, L87 (1998).

7. R. Ruffini, C. L. Bianco, P. Chardonnet, F. Fraschetti and S.-S. Xue, ApJ 555, L107 (2001).

8. R. Ruffini, C. L. Bianco, P. Chardonnet, F. Fraschetti and S.-S. Xue, ApJ 555, L113 (2001).

9. R. Ruffini, C. L. Bianco, P. Chardonnet, F. Fraschetti and S.-S. Xue, ApJ 555, L117 (2001).

10. R. Ruffini and J.R. Wilson, Phys. Rev. D12, 2959 (1975).

11. V.F. Shvartsman, Sov. Phys. JETP 33, 475 (1970).

12. B. Punsly, Black Hole Gravitohydromagnetics, Springer, 2001.

13. P. Goldreich and W.H. Julian, Ap. J. 157, 869 (1969).

14. R. Ruffini, in Proceedings of the Ninth Marcel Grossmann Meeting on General Relativity, Gurzadyan V.G., Jantzen R.T. \& Ruffini R. editors, World Scientific, Singapore (2002).

15. R. Ruffini, J. D. Salmonson, J. R. Wilson and S.-S. Xue, $A \& \& A$ 350, 334 (1999)

16. R. Ruffini, J. D. Salmonson, J. R. Wilson and S.-S. Xue, $A \& \& A$ 359, 855 (2000).

17. C. L. Bianco, R. Ruffini, S.-S. Xue, $A \mathscr{E} A$ 368, 377 (2000).

18. R. Ruffini, L. Vitagliano and S.-S. Xue, (2003) in preparation.

19. R. Ruffini, L. Vitagliano and S.-S. Xue, Phys. Lett. B559, 12 (2003).

20. R. Ruffini, L. Vitagliano and S.-S. Xue, (2003) in these proceedings.

21. C. Cherubini, R. Ruffini and L. Vitagliano, Phys. Lett. B545, 226 (2002). 


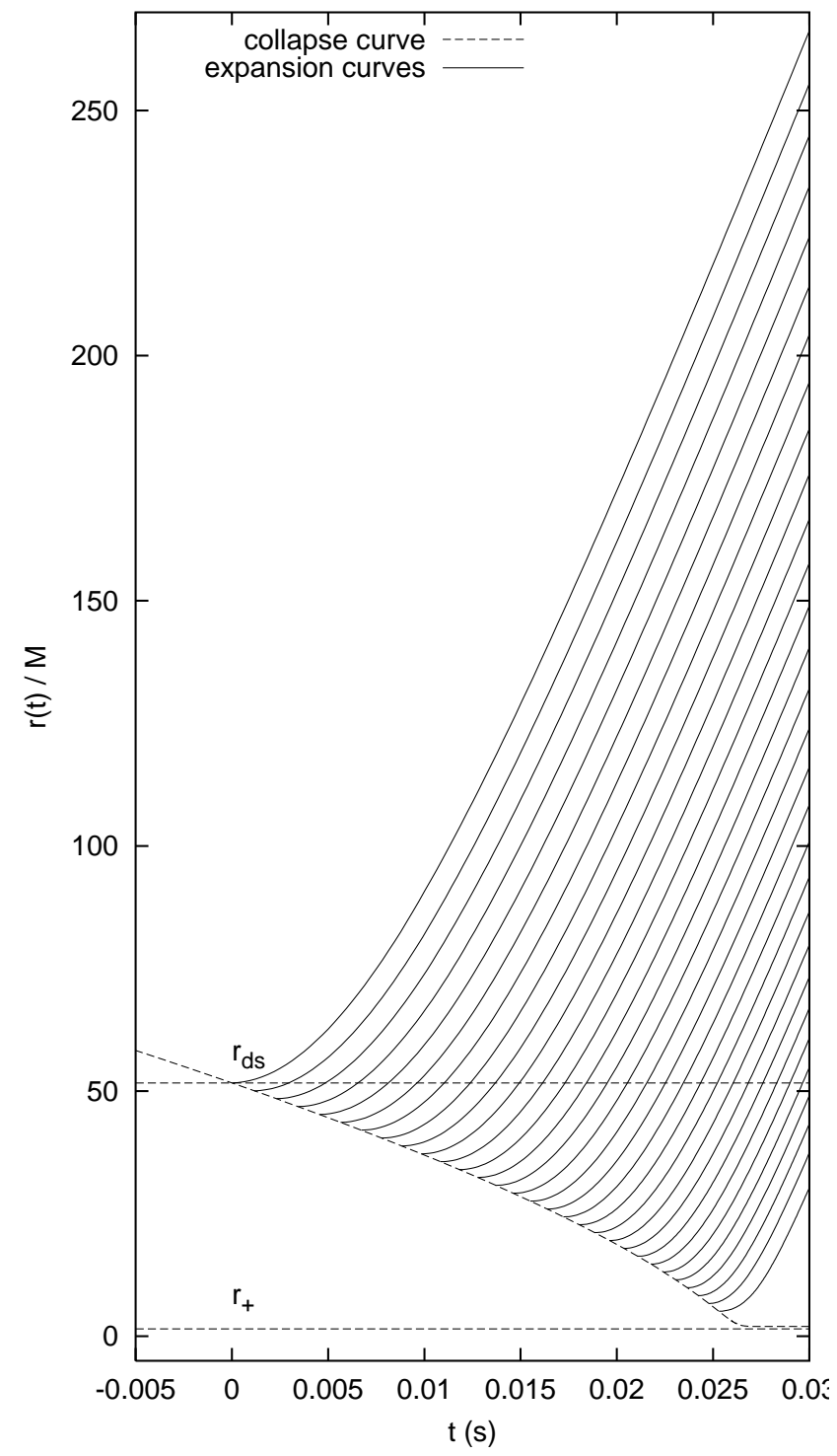

Figure 4. Collapse curve of an elettromagnetic star core (dashed line) as derived by Eq. (16) in the case $M=M_{0}=20 M_{\odot}, Q=0.1 M$ and expansion curves of plasma. 